\title{
EGFR and non-small cell lung cancer: implications for surgical practice
}

\author{
Gonzalo Varela ${ }^{1}$, Nuria M. Novoa ${ }^{2}$ \\ ${ }^{1}$ Biomedical Research Institute of Salamanca (IBSAL), Salamanca, Spain; ${ }^{2}$ Service of Thoracic Surgery, Salamanca University Hospital, Salamanca, \\ Spain \\ Correspondence to: Dr. Gonzalo Varela. Institute for Biomedical Research of Salamanca (IBSAL), Salamanca, Spain. Email: gvs@usal.es. \\ Provenance and Peer Review: This article was commissioned by the editorial office, Annals of Translational Medicine. The article did not undergo \\ external peer review. \\ Comment on: Liang W, Cai K, Chen C, et al. Society for Translational Medicine consensus on postoperative management of EGFR-mutant lung \\ cancer (2019 Edition). Transl Lung Cancer Res 2019;8:1163-73.
}

Submitted Mar 21, 2019. Accepted for publication Apr 01, 2020.

doi: $10.21037 /$ atm.2020.04.47

View this article at: http://dx.doi.org/10.21037/atm.2020.04.47

\section{Introduction}

A recently published paper from the Society for Translational Medicine (1) summarizes current multinational consensus and recommendations on postoperative management of non-small cell lung cancer (NSCLC) patients having mutant epidermal growth factor receptor (EGFR). In this report we are reviewing their recommendations and discussing the influence of the last EGFR evidences over our practice as thoracic surgeons.

\section{Early stage lung cancer therapy evolution}

Surgical therapy for NSCLC early stages has shown impressive improvements in the last 40 years (2) decreasing aggressiveness and adverse effects of the procedures. Nevertheless, a percentage of resectable tumors, including Stage I cases, have occult distant metastases at the time of surgery leading to shorter survival time. This fact leaded to development of several cisplatin-based adjuvant chemotherapy trials in resectable NSCLC. In different trials evaluating surgery plus adjuvant chemotherapy versus surgery alone, adding chemotherapy offered an absolute improvement in survival of $4-5 \%$ at 5 years $(3,4)$. Unfortunately, the inconvenience of such regimens is severe adverse effects for the patient, including death due to chemotherapy-induced toxic effects in $0.8 \%$ of the cases (5).

Current efforts are focused to identify biomarkers predicting high risk of post-surgical recurrence even in early stages of the disease. One of the most interesting discoveries in the last years is that, mainly in advances stages but also in resectable cases, a high percentage of tumor cells are positive to programmed death-ligand 1 (PD-L1) protein. When PD-L1 binds to the protein PD-1 found on T cells, it keeps $\mathrm{T}$ cells from killing the PD-L1-containing cancer cells (6). When immune checkpoint inhibitors bind to PD$\mathrm{L} 1$ and block its binding to PD-1 the immune system is released to kill cancer cells. A randomized clinical trial using pembrolizumab, a monoclonal antibody check point inhibitor blocking PD-1, demonstrated an improvement in tumor response rate and overall survival compared to standard chemotherapy in advancer NSCLC (7). Although pembrolizumab is currently first-line treatment for patients with NSCLC with PD-L1 expression $>50 \%$, the value of PD-L1 expression as a prognostic marker in resectable NSCLC is still under evaluation (8).

The role of PD-1 inhibitor nivolumab as induction treatment in resectable lung cancer has been tested in a pilot study (9). Investigators administered nivolumab to patients having untreated, surgically resectable stage I-IIIa tumors. Four weeks after first dose, 20 out of 21 cases underwent complete resection. Major pathological response was found in $45 \%$ of cases with no major side effects. A similar effect in terms of pathological response, although with a higher rate of adverse events related to the neoadjuvant therapy, has been recently published using sintilimab, another PD-1 inhibitor (10). 


\section{EGFR and NSCLC}

EGFR is a transmembrane protein that is activated by binding of its specific ligands. Upon activation by its growth factor ligands, EGFR undergoes a transition from an inactive monomeric form to an active dimeric one. EGFR dimerization stimulates its intrinsic intracellular proteintyrosine kinase (TK) activity (11).

A TK is an enzyme that can transfer a phosphate group from ATP to a protein in a cell acting as an "on" or "off" switch in many cellular functions. TK activity in the nucleus is related to cell-cycle control and properties of transcription factors and it is involved in mitogenesis (12). EGFR mutations can cause some TK to contribute to initiation or progression of cancer after entering a nonstop functional state.

The most frequent EGFR mutations are described in exon 19 and 21 in cases of adenocarcinoma histology and are in the TK domain. EGFR mutations are found more frequently in East Asian, women and non-smoking population (13). These mutations are associated with sensitivity to tyrosine-kinase inhibitors (TKIs). To date, three generations of TKIs are available in the armamentarium against EGFR-mutated tumors: erlotinib and gefitinib (first generation), afatinib (second generation) and osimertinib (third generation). Several trials comparing erlotinib, gefitinib and afatinib to chemotherapy have shown improved progression-free survival and lower toxicity but no improved overall survival in advanced (stage IIIB-IV) NSCLC patients whose tumors had an EGFR mutation (14). On the contrary, osimertinib has shown higher intracranial efficacy and better tolerability compared to gefitinib or erlotinib and longer overall survival (15).

\section{Recommendations from the society of translational medicine}

The consensus document from the Society of Translational Medicine (1) included 8 recommendations classified as "strong" according to Grading of Recommendations, Assessment, Development and Evaluations (GRADE) where the strength of recommendation is determined "by the balance between desirable and undesirable consequences of alternative management strategies, quality of evidence, variability in values and preferences, and resource use" (16). The main core of recommendations 1 to 4 are related to the convenience of detection of EGFR mutations in resected NSCLC patients and the advantages of adjuvant TKIs therapy in EGFR positive and high-risk of recurrence cases. Three of the recommendations 6,7 and 8 are referred to management of patients with actual or high-risk of postoperative metastases (brain and bone are specifically cited). Recommendation 5 specifies duration of adjuvant therapy.

Very shortly, the authors agree on the relevance of routine search for EGFR mutations in resected specimens and other driver mutations (ALK, ROS1, BRAF, KRAS, NTRK, and PDL-1) according to NCCN guidelines (17) if the conditions of the center allow for it. Doing so, recurrence risk can be stratified and, in cases with EGFR mutation adjuvant EGFR-TKIs indicated to achieve longer disease-free survival. That would be specifically relevant in cases with poor expected tolerance to conventional chemotherapy. Adjuvant therapy should last at least 2 years and patient's follow-up should be intensified to facilitate early detection of brain and bone metastases in EGFR mutated cases.

\section{Implications for surgical practice}

\section{Multidisciplinary clinical thoracic oncology boards}

Progress in diagnosis, prognosis and therapy in lung cancer makes difficult for each specialist to be updated in all topics related to the disease. This is one the of the reasons for multidisciplinary and coordinated management of lung cancer. Although not demonstrated by randomized trials and still under discussion (18), some evidence exists that multidisciplinary management improves survival after lung cancer resection (19). Besides thoracic surgeons, multidisciplinary teams must be composed by medical oncologists, radiotherapists, respiratory physicians and endoscopists, pathologists and cytopathologists and some professionals from all areas implicated in the diagnosis, inhospital and ambulatory care and follow-up of patients before, during and after treatment. If patients are discussed before therapeutic decisions, a consensus can be reached on the necessity of staging techniques, the most effective therapy and probability of relapsing of the disease. We, as thoracic surgeons must admit that our role in lung cancer management is as relevant as the role of every board member is playing.

\section{Adjuvant treatment and follow-up after complete resection in NSCLC}

Adequate surgical specimens classification and management 
in theatre is mandatory and close cooperation with pathologist to facilitate standardized gross examination and adequate histological and molecular techniques (20). Surgical teams must be efficient not only to perform radical lymph node dissection but also for specimen labeling without failures and secure transfer between the operating theatre and the pathology laboratory (21).

Correct histological and molecular evaluation of the specimens and surgical-pathological staging permits the recommendation of up-to-date adjuvant therapies. In a recently published meta-analysis (22), adjuvant treatment with TKIs after complete resection, in lung cancer patients harboring EGFR mutations, has been shown to increase disease-free survival compared to placebo and chemotherapy, and with a lower rate of adverse effects than the last. Ongoing clinical trials (23) are testing the hypothesis that nivolumab as adjuvant therapy increase disease-free survival time compared to observation and chemotherapy after surgical resection in NSCLC patients with high PD-L1 expression (23).

The role of surgical teams is not ended after lung resection. In cases of recurrence expert cooperation is needed to tumor re-biopsy without aggressive techniques. PD-L1 expression in NSCLC can change from primary to recurrence, implicating the need for re-biopsy at recurrence and the kind of therapy (24).

\section{Surgery after induction treatment with TKIs in advanced stages but resectable tumors}

The prognosis and survival of patients with Stage IV NSCLC is poor and standard management is systemic therapy including chemotherapy, targeted therapy or immunotherapy after adequate genetic and molecular testing to allow for individual treatment decisions (25). The concept of local consolidative therapy (LCT) has recently been introduced as an option to treat with radiotherapy or surgery a subset of cases with oligometastatic disease not progressing after front-line systemic therapy (26). Preliminary data suggest that in patients with oligometastatic stage IV NSCLC and without progression after front-line systemic therapy, with chemotherapy or TKIs depending on the EGFR status, LCT prolongs progression-free survival time and overall survival in comparison to maintenance therapy or observation. In Gomez's et al. series (26), only a small percentage of patients were treated with resection of the primary pulmonary tumor as a fundamental component of LCT but in Zhang et al. (27), 86 stage IV NSCLC patients underwent pulmonary resection plus local therapy for metastatic lesion in most cases, followed by adjuvant therapy. For the whole series, the estimated 3 -year overall survival was $42.2 \%$. To note is that EGFR patients treated with TKIs had significantly better survival compared to those with adjuvant chemotherapy.

Many new and promising pathways are still to be explored in the treatment of localized and advanced stages of NSCLC. Surgery will continue to be a fundamental part of therapy but never alone but in coordination with other fundamental branches of multi-disciplinary teams.

\section{Acknowledgments}

Funding: None.

\section{Footnote}

Conflicts of Interest: Both authors have completed the ICMJE uniform disclosure form (available at http://dx.doi. org/10.21037/atm.2020.04.47). GV serves as an unpaid editorial board member of Annals of Translational Medicine from Jun 2019 to May 2024. NMN serves as an unpaid editorial board member of Shanghai Chest from Aug 2018 to Jul 2020.

Ethical Statement: The authors are accountable for all aspects of the work in ensuring that questions related to the accuracy or integrity of any part of the work are appropriately investigated and resolved.

Open Access Statement: This is an Open Access article distributed in accordance with the Creative Commons Attribution-NonCommercial-NoDerivs 4.0 International License (CC BY-NC-ND 4.0), which permits the noncommercial replication and distribution of the article with the strict proviso that no changes or edits are made and the original work is properly cited (including links to both the formal publication through the relevant DOI and the license). See: https://creativecommons.org/licenses/by-nc-nd/4.0/.

\section{References}

1. Liang W, Cai K, Chen C, et al. Society for Translational Medicine consensus on postoperative management of EGFR-mutant lung cancer (2019 Edition). Transl Lung Cancer Res 2019;8:1163-73. 
2. Novello S, Asamura H, Bazan J, et al. Early stage lung cancer: Progress in the last 40 years. J Thorac Oncol 2014;9:1434-42.

3. Pignon JP, Tribodet H, Scagliotti GV, et al. Lung adjuvant cisplatin evaluation: a pooled analysis by the LACE Collaborative Group. J Clin Oncol 2008;26:3552-9.

4. Burdett S, Pignon JP, Tierney J, et al Adjuvant chemotherapy for resected early-stage non-small cell lung cancer. Cochrane Database Syst Rev 2015;(3):CD011430.

5. Arriagada R, Bergman B, Dunant A, et al. Cisplatinbased adjuvant chemotherapy in patients with completely resected non-small-cell lung cancer. $\mathrm{N}$ Engl J Med 2004;350:351-60.

6. Owen D, Chaft JE. Immunotherapy in surgically resectable non-small cell lung cancer. J Thorac Dis 2018;10:S404-11.

7. Reck M, Rodriguez-Abreu D, Robinson AG, et al. Pembrolizumab versus Chemotherapy for PD-L1Positive Non-Small-Cell Lung Cancer. N Engl J Med 2016;375:1823-33.

8. D'Arcangelo M, D'Incecco A, Ligorio C, et al.

Programmed death ligand 1 expression in early stage, resectable non-small cell lung cancer. Oncotarget 2019;10:561-72.

9. Forde PM, Chaft JE, Smith KN, et al. Neoadjuvant PD-1 blockade in resectable lung cancer. $\mathrm{N}$ Engl J Med 2018;378:1976-86.

10. Gao S, Li N, Gao S, et al. Neoadjuvant PD-1 inhibitor (Sintilimab) in NSCLC. J Thorac Oncol 2020. pii: S15560864(20)30057-5.

11. Westerfield JM, Barrera FN. Membrane receptor activation mechanisms and transmembrane peptide tools to elucidate them. J Biol Chem 2020;295:1792-814.

12. Paul MK, Mukhopadhyay AK. Tyrosine kinase. Role and significance in Cancer. Int J Med Sci 2004;1:101-15.

13. Grigoriu B, Berghmans T, Pascale Meert AP. Management of EGFR mutated nonsmall cell lung carcinoma patients. Eur Respir J 2015;45:1132-41.

14. Greenhalgh J, Dwan K, Boland A, et al. Firstline treatment of advanced epidermal growth factor receptor (EGFR) mutation positive non-squamous nonsmall cell lung cancer. Cochrane Database Syst Rev 2016;(5):CD010383.

15. Soria JC, Ohe Y, Vansteenkiste J, et al. Osimertinib in Untreated EGFR-Mutated Advanced Non-Small-Cell Lung Cancer. N Engl J Med 2018;378:113-25.

16. Guyatt GH, Oxman AD, Kunz R, et al. Going from evidence to recommendations. BMJ 2008;336:1049-51.
17. Ettinger DS, Wood DE, Aggarwal C, et al. NCCN Guidelines Insights: Non-Small Cell Lung Cancer, Version 1.2020. J Natl Compr Canc Netw 2019;17:1464-72.

18. Fleissig A, Jenkins V, Catt $S$, et al. Multidisciplinary teams in cancer care: are they effective in the UK? Lancet Oncol 2006;7:935-43.

19. Tamburini N, Maniscalco P, Mazzara S, et al. Multidisciplinary management improves survival at 1 year after surgical treatment for non-small-cell lung cancer: a propensity score-matched study. Eur J Cardiothorac Surg 2018;53:1199-204.

20. Mansuet-Lupo A, Filaire M, Chaffanjon P, et al. Guidelines for the macroscopic management of surgically resected lung carcinoma. Ann Pathol 2019;39:425-32.

21. Osarogiagbon RU, Hilsenbeck HL, Sales EW, et al. Improving the pathologic evaluation of lung cancer resection specimens. Transl Lung Cancer Res 2015;4:432-7.

22. Cheng H, Li XJ, Wang XJ, et al. A meta-analysis of adjuvant EGFR-TKIs for patients with resected non-small cell lung cancer. Lung Cancer 2019;137:7-13.

23. Chaft, JE, Dahlberg SE, Gerber DE, et al. EA5142 adjuvant nivolumab in resected lung cancers (ANVIL): The newest study in the ALCHEMIST platform. J Clin Oncol 2017. doi: 10.1200/JCO.2017.35.15_suppl. TPS8575.

24. Lacour M, Hiltbrunner S, Lee SY, et al. Adjuvant chemotherapy increases programmed death-ligand 1 (PDL1) expression in non-small cell lung cancer recurrence. Clin Lung Cancer 2019;20:391-6.

25. Planchard D, Popat S, Kerr K, et al. Metastatic non-small cell lung cancer: ESMO Clinical Practice Guidelines for diagnosis, treatment and follow-up. Ann Oncol 2018;29:iv192-iv237.

26. Gomez DR, Tang C, Zhang J, et al. Local consolidative therapy vs. maintenance therapy or observation for patients with oligometastatic Non-Small-Cell Lung Cancer: Longterm results of a multi-institutional, Phase II, randomized study. J Clin Oncol 2019;37:1558-65.

27. Zhang C, Wang L, Li W, et al. Surgical outcomes of stage IV non-small cell lung cancer: a single-center experience. J Thorac Dis 2019;11:5463-73.

Cite this article as: Varela G, Novoa NM. EGFR and nonsmall cell lung cancer: implications for surgical practice. Ann Transl Med 2020;8(17):1115. doi: 10.21037/atm.2020.04.47 